\title{
Capping layer effects in the structure and composition of Co nanoparticle ultrathin films
}

\author{
J. Arbiol, ${ }^{a)}$ F. Peiró, and A. Cornet \\ EME, Departament d'Electrònica, Universitat de Barcelona, C/ Martí i Franquès 1, \\ E-08028 Barcelona, Spain \\ C. Clavero, A. Cebollada, and G. Armelles \\ Instituto de Microelectrónica de Madrid (CNM-CSIC), Isaac Newton 8-PTM, \\ E-28760 Tres Cantos, Madrid, Spain \\ Y. Huttel \\ Instituto de Ciencia de Materiales de Madrid (ICMM-CSIC), 28029 Cantoblanco, Madrid, Spain
}

(Received 27 September 2004; accepted 26 November 2004; published online 12 January 2005)

In this work, we present the correlation of the magnetic and structural properties of Co nanoparticles deposited by sputtering on $\mathrm{Si}_{3} \mathrm{~N}_{4}$ substrates at different temperatures, and covered with different capping layers, two insulators, $\mathrm{AlN}$ and $\mathrm{MgO}$, and a metal, Pt. High-resolution transmission electron microscopy shows the formation of $\mathrm{CoPt}_{3}$ and $\mathrm{Co}_{2} \mathrm{~N}$, for the Pt and AlN capping layers, respectively, giving to a significant change of the magnetic behavior. When using a cap of $\mathrm{MgO}$, energy-filtered transmission electron microscopy shows an oxidized shell covering the Co nanoparticles with thickness decreasing as the deposition temperature increases, explaining the changes in the magnetic response induced by the $\mathrm{MgO}$ capping layer. (C) 2005 American Institute of Physics. [DOI: 10.1063/1.1854746]

Synthesis of magnetic nanoparticles embedded in a dielectric or metal matrix is currently of great interest due to their application in the fabrication of ultra-high-density recording media ${ }^{1-3}$ and magneto-optical sensors and devices with tailored properties. ${ }^{3-5}$ Several studies have been already made in order to determine the magnetic response of these nanoparticles, by studying their transverse and polar magneto-optical Kerr signal, ${ }^{6-8}$ or even theorizing and modeling how the magnetic dipolar interactions modify the superparamagnetic relaxation rate. ${ }^{9,10}$

The nature of the different capping layers used to protect magnetic nanoparticles in ultrathin film against oxidation may induce strong modifications of their magnetic properties. For example, a Pt capping layer may induce the magnetic connection of otherwise magnetically disconnected $\mathrm{Fe}$ islands grown on $c$-sapphire substrates ${ }^{7,11}$ while deposition of $\mathrm{MgO}$ as a capping layer on the same system produces no modification of the magnetic properties, as $\mathrm{MgO}$ is not magnetically polarizable. ${ }^{7}$ Moreover, the use of AlN as capping layer may induce the opposite effect than Pt, as it may lead to the magnetic disconnection between ferromagnetic Co particles grown on AlN thin films. ${ }^{12}$

However, while the effects of these capping layers on the magnetic properties of the nanoparticle system have already been correlated with the intrinsic properties of the cap or with the nature of the deposition technique, a detailed structural and chemical characterization able to give a picture at the atomic level of the different caps effects with high resolution microscopy techniques is still missing. The aim of this letter is to carry out such a characterization in a set of Co nanoparticle ultrathin films by high resolution transmission electron microscopy (HRTEM) and energy filtered transmission electron microscopy (EFTEM) analysis.

\footnotetext{
a) Author to whom correspondence should be addressed; electronic mail: arbiol@el.ub.es
}

The samples were grown in an ultrahigh vacuum multichamber equipped with sputtering and laser ablation facilities (base pressure below $10^{-9} \mathrm{mbar}$ ). Co deposition was performed at temperatures ranging from room temperature (RT) to $700{ }^{\circ} \mathrm{C}$ on a $\mathrm{Si}_{3} \mathrm{~N}_{4}$ substrate. Subsequently, $\mathrm{MgO}, \mathrm{Pt}$, and AlN were grown as capping layers using different techniques. The structural characterization was carried out by HRTEM and EFTEM. In all cases, the magnetic response of the nanoparticles was studied by in situ transversal Kerr loops before and after the deposition of the capping layers.

We first show the HRTEM results for the $\mathrm{MgO}$ cap. Samples with $2 \mathrm{~nm}$ Co nominal thicknesses were deposited at different temperatures $\left(200,400,550\right.$, and $\left.700{ }^{\circ} \mathrm{C}\right)$ and a 3 $\mathrm{nm}$ thick $\mathrm{MgO}$ capping layer was deposited at $\mathrm{RT}$ by laser ablation from a $\mathrm{MgO}$ target. Bright field TEM (BFTEM) revealed a granular morphology for all deposition temperatures, with a small reduction of the particle concentration as Co deposition temperature increases. Figures 1(a) and 1(b) show BFTEM micrographs of the sample grown at $550{ }^{\circ} \mathrm{C}$, representative of the series. We found by HRTEM that Co mainly crystallized as Co metal nanoparticles in its hexagonal metallic phase and no interaction with $\mathrm{MgO}$ layer nor nitridation from the inner substrate was detected, although in the as-grown sample (RT) we also found a minor percentage of nanoparticles in oxidized phases such as $\mathrm{Co}_{3} \mathrm{O}_{4}$. From TEM measurements, the mean diameter of the Co nanoparticles ranged from $5.0 \pm 0.6 \mathrm{~nm}$ in the sample grown at $200{ }^{\circ} \mathrm{C}$ to $8.3 \pm 1.6 \mathrm{~nm}$ in the sample grown at $700{ }^{\circ} \mathrm{C}$.

Samples were also studied by electron energy loss spectroscopy (EELS). By selecting the $60 \mathrm{eV}$ energy loss peak, which corresponds to the energy of $M_{2,3}$ Co transitions, we obtained several EFTEM images [Fig. 1(c)] of the sample grown at $550{ }^{\circ} \mathrm{C}$, in the same regions previously studied by BFTEM [Fig. 1(a)]. We observed an increase of the mean diameter of Co nanocrystals measured from EFTEM, in comparison with that measured from $\mathrm{BF}$ micrographs, for 

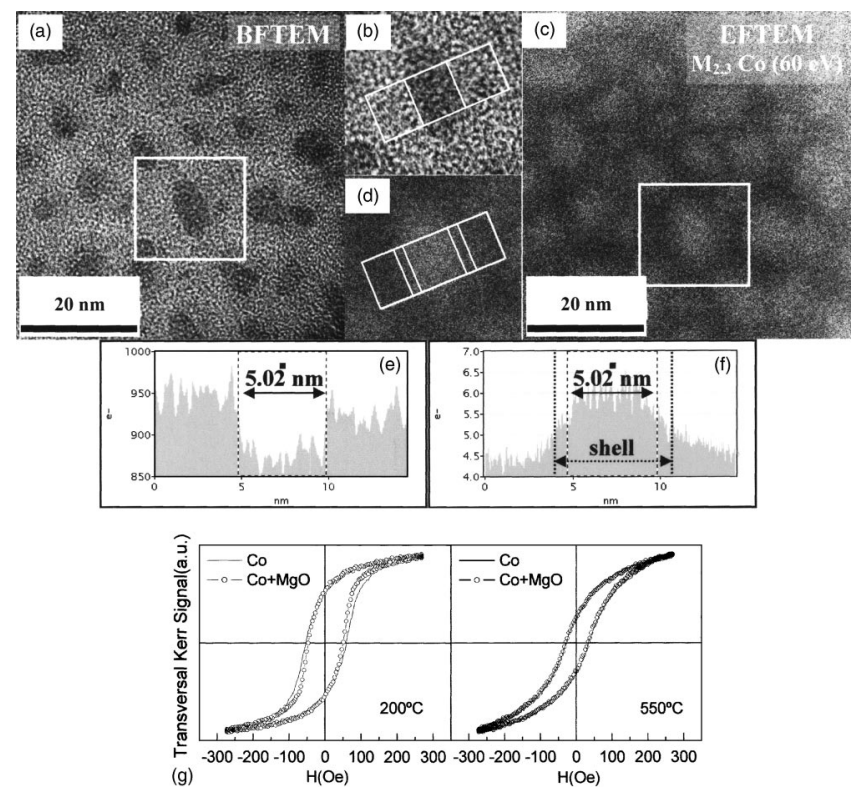

FIG. 1. (a) BFTEM image of the Co nanoclusters in a $\mathrm{MgO} / \mathrm{Co} / \mathrm{Si}_{3} \mathrm{~N}_{4}$ sample grown at $550{ }^{\circ} \mathrm{C}$; (b), (e) profile analysis of the selected Co nanocluster in (a); (c) energy filtered TEM image of the same region; (d), (f) profile analysis of the selected Co nanocluster in (c); (g) in situ transversal Kerr loops before (continuous line) and after (circles) the $\mathrm{MgO}$ capping layer deposition in samples grown at 200 and $550^{\circ} \mathrm{C}$.

sample grown at low temperature (LT), while there was no variation for diameters measured with both imaging modes for sample grown at high temperature (HT). As BFTEM images enhance the contrast of crystalline structures with respect to amorphous areas (diffraction contrast) while EFTEM images contrast is directly proportional to the number of atoms of the selected element in the sample, we can conclude, that Co nanoparticles have a crystalline nucleus (as revealed by BFTEM) as well as a surrounding amorphous Co shell with a thickness that decreases as the deposition temperature increases (EFTEM images). Analyzing the profile intensity of the EFTEM Co signal [Figs. 1(d) and 1(f)], we observe a dramatic decrease in the region corresponding to the shell. This latter result shows that the Co density is lower in the borders of the clusters with respect to the crystalline nucleus, indicating that there must be a lighter element coexisting with the Co atoms. Our EELS results suggest that an amorphous $\mathrm{Co}_{x} \mathrm{O}_{y}$ oxidized shell should exist encapsulating the Co crystalline nanoparticles nucleus in the samples grown at lower temperature.

The appearance of the mentioned amorphous crust and the fact that its thickness decreases as the Co deposition temperature increases can be tentatively explained as an effect of the crystalline quality of the nanoparticles. Co nanoparticles grown at LT have worse crystalline quality, favoring the reaction of Co atoms with the $\mathrm{MgO}$ capping layer. The better crystalline quality observed by TEM at HT deposition makes more difficult the interdiffusion of Co atoms in the capping layer.

These results explain the change of the magnetic response of the nanoparticles observed in the in situ transversal Kerr loops [Fig. 1(g)]. At lower Co deposition temperatures a decrease in the coercitive field has been observed after the deposition of the $\mathrm{MgO}$ capping layer, due to the formation of the aforementioned amorphous oxidized shell and the consequent reduction of the magnetic size of the nanoparticles. At Downloaded 05 Nov 2009 to 161.111.235.169. Redistribution subject
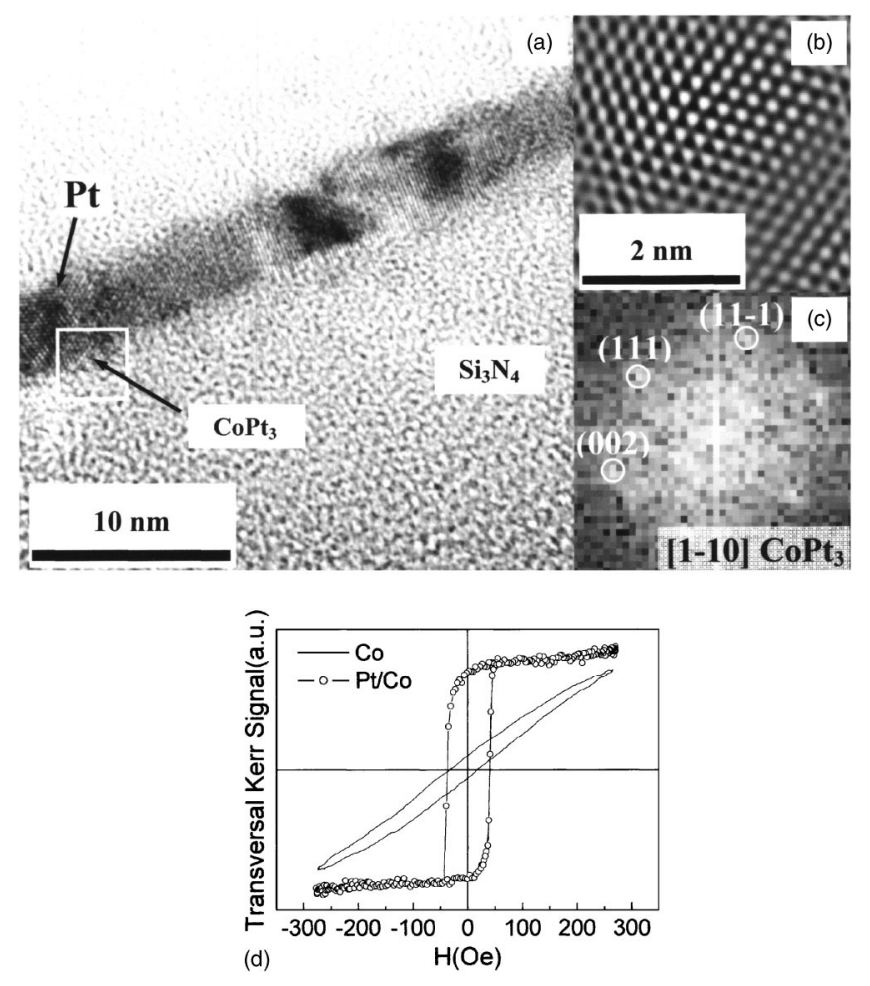

FIG. 2. (a) HRTEM micrograph of a Pt/Co/ $\mathrm{Si}_{3} \mathrm{~N}_{4}$ sample grown at $400{ }^{\circ} \mathrm{C}$; (b) HRTEM image of the $\mathrm{CoPt}_{3}$ nanocluster squared in (a); (c) FFT of the selection in (a); (d) in situ transversal Kerr loops before (continuous line) and after (circles) the Pt capping layer deposition.

higher Co deposition temperatures no effect of the capping layer is observed in the transversal loops because, as noticed, the crystalline Co core is less affected by the $\mathrm{MgO}$ capping.

A second series of samples, with Co nominal thickness of $1 \mathrm{~nm}$ grown at different deposition temperatures were studied. Here a $2.5 \mathrm{~nm}$ thick Pt cap was deposited at RT by sputtering from a Pt target, warranting the isolated Co particle formation. HRTEM revealed that the capping layer was composed of $\mathrm{Pt}$ in its metal cubic phase for all growth temperatures. Moreover, we found that at RT the sputtered Co crystallized as metal hexagonal-phase nanoparticles. However, when increasing the Co growth temperature, we detected Pt diffusion towards the Co nanoparticles inner layer, inducing the formation of different $\mathrm{Co}-\mathrm{Pt}$ binary alloys (such as $\mathrm{CoPt}_{3}$ at $400{ }^{\circ} \mathrm{C}$ ), and even metal oxide complex phases (such as $\mathrm{CoPt}_{3} \mathrm{O}_{6}$ ) for higher Co deposition temperatures $\left(700{ }^{\circ} \mathrm{C}\right)$, due to the enhanced Pt diffusivity from the capping layer towards the Co nanocrystals. ${ }^{13}$ As an example, in Fig. 2(a), we show a HRTEM micrograph of the $\mathrm{Pt} / \mathrm{Co} / \mathrm{Si}_{3} \mathrm{~N}_{4}$ sample grown at $400{ }^{\circ} \mathrm{C}$.

In situ transversal Kerr loops were measured before and after the Pt capping layer deposition, in Fig. 2(d) we display for example the hysteresis loops corresponding to the sample grown at $400{ }^{\circ} \mathrm{C}$. Before the capping layer we observe a rounded hysteresis loop with no saturation at the maximum applied field, indicating that the nanoparticles do not coalesce and they are not well magnetically connected. After the deposition of the Pt capping a more squared hysteresis loop is observed revealing the magnetic connection between the Co nanoparticles mediated by the polarized $\mathrm{Pt}$ as observed elsewhere. ${ }^{8,11}$ This behavior is observed in all the samples independently of their deposition temperature, suggesting that the formation of mixed $\mathrm{Co}_{x} \mathrm{Pt}_{y}$ phases has not affected to AlP license or copyright; see http://apl.aip.org/apl/copyright.jsp 

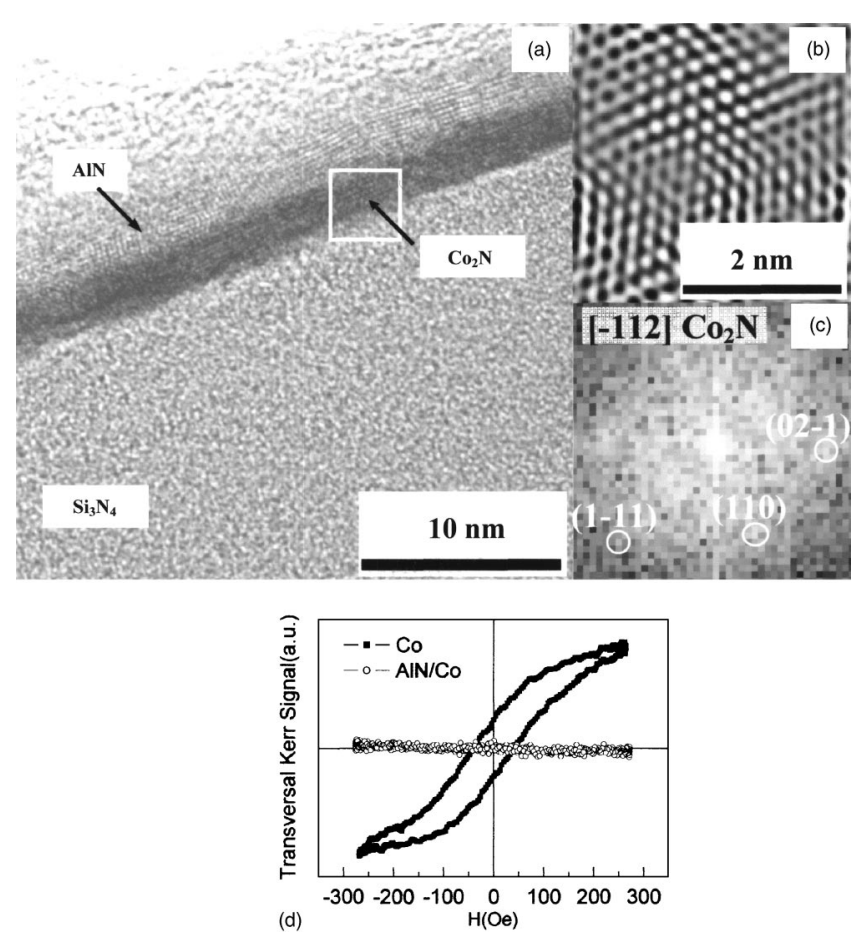

FIG. 3. (a) HRTEM micrograph of an as-grown $A l N / C o / \mathrm{Si}_{3} \mathrm{~N}_{4}$ sample (RT); (b) HRTEM image of the $\mathrm{Co}_{2} \mathrm{~N}$ nanocluster squared in (a); (c) FFT of the selection in (a); (d) in situ transversal Kerr loops before (squares) and after (circles) the AlN capping layer deposition.

the magnetic interconnection of the nanoparticles due to the polarization of Pt capping.

A similar series of samples was studied in the case of an AlN capping layer. In this case the nominal Co thickness was $2 \mathrm{~nm}$ for all the samples grown at different temperatures, and $6 \mathrm{~nm}$ of AlN were grown at $400{ }^{\circ} \mathrm{C}$ by reactive sputtering using an $\mathrm{Al}$ target and an $\mathrm{Ar}^{+} \mathrm{N}_{2}$ plasma. As observed in Fig. 3 , the upper capping layer conserves its AlN structure, but HRTEM measurements reveal that even at RT, a strong Co nitridation occurred, finding $\mathrm{Co}_{2} \mathrm{~N}$ compounds instead of metallic Co nanoparticles [Figs. 3(b) and 3(c)]. At higher temperatures, 400 and $700{ }^{\circ} \mathrm{C}$, we also found a strong Co nitridation and compounds as $\mathrm{Co}_{2} \mathrm{~N}$ and even $\mathrm{CoN}$ were found, which implies a strongest diffusion of nitrogen.

The effect of the AlN capping in the magnetic behavior of the system is very strong. In situ Kerr transversal loops were measured before and after the deposition of the AlN capping [Fig. 3(d)] in a sample grown at $400{ }^{\circ} \mathrm{C}$. A ferromagnetic hysteresis loop was obtained after the Co deposition, corresponding to a system with Co nanoparticles magnetically connected but after the AlN capping deposition the magnetic signal disappears. In order to clarify the cause of this disappearance a sample was exposed to an $\mathrm{Ar}+\mathrm{N}_{2}$ plasma after the Co deposition, and the magnetic signal also disappeared. Therefore we can conclude that the exposition of the Co nanoparticles to a $\mathrm{N}_{2}$ plasma gives rise to a strong nitridation and the disappearance of the magnetic behavior of this system.

In these two last cases, deposition of Pt and AlN caps induced the formation of nonferromagnetic Co alloys $\left(\mathrm{Co}_{x} \mathrm{Pt}_{1-x}\right)$ and compounds (Co nitrides) which correlate well with the changes in the magnetic properties observed in previous works.

It is therefore concluded that, when Pt is deposited as capping layer the polarization of Pt next to the Co nanoparticles give rise to the magnetic connection between the islands, and diffusion of Pt into the Co nanoparticles is observed at HT. The AlN capped samples show a strong nitridation of $\mathrm{Co}$ and the magnetic signal consequently disappears. In the former samples, interface diffusion is minimized by selecting the appropriate deposition temperature. Finally, the $\mathrm{MgO}$ has been presented as a good capping option, since little interaction between this material and the Co nanoparticles magnetic active layer has been found. A small decrease of the coercitive field before the capping layer deposition is observed, related to a decrease of the magnetic particle size, in samples grown at LT. This fact has been explained by the use of HRTEM in combination with EFTEM. The presence of an amorphous crust surrounding the Co nanoparticles with decreasing thickness as the deposition temperature increase has been inferred.

This work has been funded by the Spanish Project No. MAT2002-04484-C03-02. Y.H. acknowledges the Consejo Superior de Investigaciones Científicas (CSIC) and Ramón y Cajal Program for financial support.

${ }^{1}$ V. F. Puntes, P. Gorostiza, D. M. Aruguete, N. G. Bastus, and A. P. Alivisatos, Nat. Mater. 3, 263 (2004).

${ }^{2}$ S. Sun and C. B. Murray, J. Appl. Phys. 85, 4325 (1999).

${ }^{3}$ S. Sun, C. B. Murray, D. Weller, L. Folks, and A. Moser, Science 287, 1989 (2000).

${ }^{4}$ M. Jamet, W. Wernsdorfer, C. Thirion, D. Mailly, V. Dupuis, P. Mélinon, and A. Pérez, Phys. Rev. Lett. 86, 4676 (2001).

${ }^{5}$ V. F. Puntes, K. M. Krishnan, and A. P. Alivisatos, Science 291, 2115 (2001).

${ }^{6}$ J. L. Menéndez, B. Bescós, G. Armelles, R. Serna, J. Gonzalo, R. Doole, A. K. Petford-Long, and M. I. Alonso, Phys. Rev. B 65, 205413 (2002).

${ }^{7}$ E. Navarro, Y. Huttel, C. Clavero, A. Cebollada, and G. Armelles, Phys. Rev. B 69, 224419 (2004).

${ }^{8}$ B. Kalska, J. J. Paggel, and P. Fumagalli, J. Appl. Phys. 92, 7481 (2002).

${ }^{9}$ F. Luis, F. Petroff, J. M. Torres, L. M. García, J. Bartolomé, J. Carrey, and A. Vaurès, Phys. Rev. Lett. 88, 217205 (2002).

${ }^{10}$ D. J. Sellmyer, M. Yu, and R. D. Kirby, Nanostruct. Mater. 12, 1021 (1999).

${ }^{11}$ E. Navarro, Y. Huttel, C. Clavero, G. Armelles, and A. Cebollada, Appl. Phys. Lett. 842139 (2004).

${ }^{12}$ Y. Huttel, H. Gómez, C. Clavero, G. Armelles, A. Cebollada, E. Navarro, M. Ciria, L. Benito, J. I. Arnaudas, and A. J. Kellock, J. Appl. Phys. 96, 1666 (2004).

${ }^{13}$ V. Parasote, O. Ersen, V. Pierron-Bohnes, C. Ulhaq-Bouillet, D. Spor, J. Arabski, and M. C. Cadeville, Diffusion Defect Data--Solid State Data 194-199, 1357 (2001). 